Réapprendre à habiter ici et entre nous : une éducation centrée sur les lieux et la communauté

Tom Berryman

\author{
(2) OpenEdition \\ Journals \\ Édition électronique \\ URL : http://journals.openedition.org/ere/4200 \\ DOI : $10.4000 /$ ere.4200 \\ ISSN : 2561-2271 \\ Éditeur \\ Centr'ERE
}

Référence électronique

Tom Berryman, « Réapprendre à habiter ici et entre nous : une éducation centrée sur les lieux et la communauté », Éducation relative à l'environnement [En ligne], Volume 5 | 2005, mis en ligne le 20 novembre 2005, consulté le 21 février 2020. URL : http://journals.openedition.org/ere/4200 ; DOI $10.4000 /$ ere. 4200

Ce document a été généré automatiquement le 21 février 2020. 


\title{
Réapprendre à habiter ici et entre nous : une éducation centrée sur les lieux et la communauté
}

\author{
Tom Berryman
}

1 Cet article explore la question de l'éducation relative à l'environnement centrée sur la communauté et sur les lieux. Les discours et les pratiques à cet effet sont actuellement en forte émergence. Or qu'en est-il? Que veut-on dire lorsqu'il est question d'une éducation centrée sur les lieux et sur la communauté ? Quelles sont ses caractéristiques et en quoi sont-elles différentes de celles d'autres approches. Le thème de ce volume, «Cultures et territoires : ancrages pour une éducation relative à l'environnement », est nettement en résonance avec les questions de communautés et de lieux. Ce thème offre donc, une nouvelle fois, l'occasion de chercher à faire le point sur ces questions ${ }^{1}$.

\section{Un prélude par notre « être-au-monde » ... ou par notre « monde vécu »}
Circonstance! Circum stantia! Ce sont les choses muettes tout autour de nous. [...] Nous allons aveuglément parmi elles, notre regard fixé sur nos entreprises vagues et lointaines, embarqués vers la conquête de villes schématiques tout au
loin.
José Ortega Y. Gasset (1914, p. 41) - Meditaciones del Quijote

2 Les relations des êtres humains avec le monde qui les entoure occupent une place centrale de la philosophie. Ces notions de relations à l'environnement et d'êtres environnés mettent en tension les «êtres» et le «monde» et incluent l'altérité humaine en tant que composante du monde où vit chaque être. Par exemple, dans ce que l'on nomme souvent la pensée occidentale ou encore l'imaginaire occidental, et que 
l'on fait généralement remonter à la Grèce antique, le clivage entre "nature " et "culture" occupe une place centrale et presque fondatrice qui continue de nous hanter. De cette rupture est née toute une série de conduites et d'institutions importantes, tellement que Ralph Waldo Emerson (1856), dans Essays and English Traits, avance l'idée que toutes les institutions d'un peuple sont déterminées par sa vision de la nature. Évidemment, toutes les cultures ne partagent pas la même lecture du rapport au monde non plus que les mêmes oppositions binaires, les mêmes clivages. Toutefois, comme le suggère Lévi-Strauss, les oppositions binaires sont parmi les « données » les plus « communes » et les «plus riches dans la pensée humaine et dans les sociétés » et pour lui, « le binarisme n'est donc dans l'esprit que parce qu'il est déjà dans le corps » (Lévi-Strauss, 2003). Ainsi, que ce soit dans le monisme ou le dualisme, on couple et on clive assez souvent, et ce, dans toutes les cultures. Philosophiquement, la phénoménologie tente une certaine réconciliation en nous parlant de "l'être-aumonde » et du «monde vécu ». Pourtant, presque en un clin d'œil, la phénoménologie offre encore un couplage de la réalité avec deux versants.

"L'être-au-monde » serait-il en effet le "versant humain " du "monde vécu » qui serait quant à lui le « versant milieu » d'une réalité que l'on peut voir comme unitaire ou comme réellement duale? Parlons dès lors du «versant humain » et du "versant milieu » d'une réalité ou d'un regard porté sur la réalité. Ceci nous ramène à des questions de base pour des éducateurs. Quelle conception de l'être humain, quelle conception de la nature et quelle conception des liens entre les deux animent notre philosophie de l'éducation, notre approche pédagogique et nos pratiques éducatives ?²

Voyons alors comment les constructions sociales que sont les disciplines ou les champs de savoirs peuvent décliner ces versants et quels regards elles invitent à porter par ces déclinaisons. Comme exercice de dé-réification, à titre exploratoire non exhaustif, la figure 1 propose quelques mots clefs associés à différents regards sur le couplage « être humain » et "milieu $»^{3}$. Ainsi, dans sa vision du monde ou sa cosmologie, un regard formé par la biologie ou la macro-écologie pourrait décliner le monde avec un « versant milieu » vu comme biosphère et un "versant humain » vu en tant qu'Homo sapiens, l'espèce. Un regard macro-économique pourrait voir du capital partout, avec sur le "versant humain » de sa totalité, du capital humain, et sur le «versant milieu», du capital naturel.

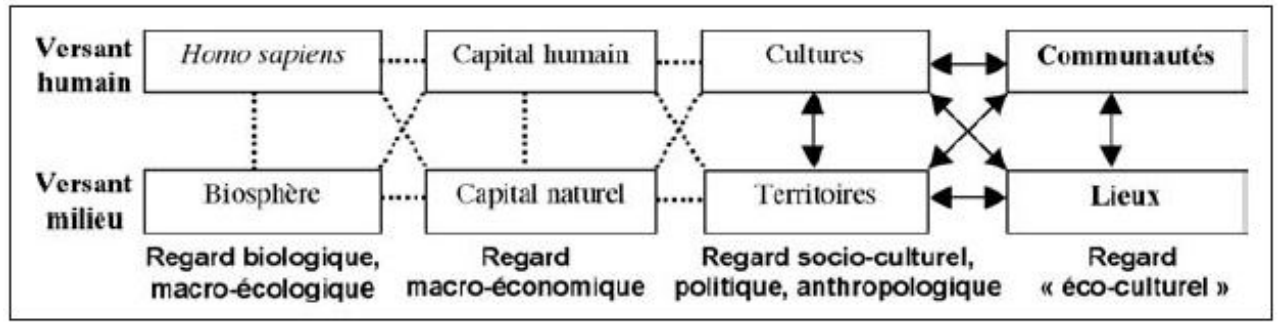

Figure 1 : Carte exploratoire des deux versants de différents regards sur le monde

5 Les entrées de la figure 1 pourraient se multiplier pour y inclure différents regards. L'entrée par le clivage "culture / nature » devrait y figurer presque en tête de liste, comme icône et probablement de manière transversale, tellement cette rupture, socialement construite, traverse des champs de savoirs. Historiquement, avec la montée de l'empirisme, du rationalisme et du positivisme, incarnés notamment par des figures de proue telles Francis Bacon (1561-1626), René Descartes (1596-1650) et 
Auguste Comte (1798-1857), on voit dans la même veine s'affirmer le couple « sujet / objet " qui pourrait lui aussi occuper une place centrale. Plus près de nous dans le temps, au début du XXe siècle, le débat étasunien autour de la construction d'un barrage dans la vallée d'Hetch Hetchy oppose Gifford Pinchot (1865-1946) du Service américain des forêts et John Muir (1838-1914) du Club Sierra. Ce débat oppose en quelque sorte un couplage qu'on dit utilitariste, conservationniste, soit le couple "gestionnaire / ressource» incarné par Pinchot, à un couplage qu'on dit "préservationniste» soit un couple « habitant / nature sauvage » ou encore «home / wilderness" incarné par Muir. Dans ce cas, le couple "gestionnaire / ressource" domina le couple «habitant / nature sauvage» et imposa sa logique environnementale : le barrage fut construit.

6 À titre exploratoire, plaçons sur cette figure le thème de ce volume: cultures et territoires. On peut supposer qu'il s'agit d'un certain regard socioculturel, politique et anthropologique et que l'idée de «cultures» correspond davantage au "versant humain » et que " territoires » correspond davantage au "versant milieu ». Il existe des liens étroits entre ces notions et les réalités qu'elles désignent, d'où les liens entre les boîtes. De même, on peut aussi situer le couple « communautés » et «lieux » sur cette figure et le nommer, à titre exploratoire, de regard « écoculturel ».

7 Si Fernand Dumont (1968) fait de la culture le «lieu de l'homme", on peut aussi inverser la rhétorique et avancer les lieux de l'homme comme un des socles de la culture. Une culture s'incarne dans des lieux. Elle est l'écho de communautés et elle est en résonance avec ces lieux. Pensons seulement, à titre d'exemples contrastés, aux lieux amazoniens de la culture Kayapo par rapport aux lieux désertiques étasuniens de la culture Hopi. Or, la perspective globalisante et planétaire tend à occuper de plus en plus de place et à occulter les dimensions charnelles et incarnées localement. Malgré cette perspective, le corps et ses assises existent toujours en un lieu et au sein d'une communauté. On ne peut qu'avoir l'illusion d'une vie non incarnée en des lieux et au sein de communautés, au sein de milieux de vie. De plus, ces lieux et l'expérience de ces lieux font partie de la constitution même de nos êtres, de notre formation, de notre écoformation (Pineau, 1999) et de notre éco-ontogenèse (Berryman, 2002a, 2003). Enfin, cette résonance du milieu dans la constitution de notre être n'agit pas qu'au seul niveau personnel, mais aussi comme partie prenante sociale, communautaire et culturelle, dans la culture matérielle et symbolique.

\section{Émergences totalisantes, clivages et désenchantements}

Pour quelle destination, pour quelle destinée éduquons-nous nos enfants? Malgré tout le discours à la mode au sujet du multiculturalisme, le fait demeure que l'éducation moderne a grandement contribué à la destruction de cultures locales pratiquement partout. La localité n'a pas de place dans le curriculum moderne. Les abstractions, les connaissances généralisées et la technologie en ont une. L'éducation est devenue une grande force d'homogénéisation minant le 
savoir local, les langues autochtones et la confiance en soi de peuples localisés. L'éducation est devenue un auxiliaire de l'économie commerciale. Elle s'est livrée aux forces de la croissance et du développement qui, pourvu que je sache, constituent un effort visant à rendre le monde sécuritaire pour le grand capital. David Orr (1994, p. 129) - Earth in Mind, traduction libre

Pour le meilleur comme pour le pire, l'histoire ou les histoires de l'éducation relative à l'environnement et de l'environnementalisme sont étroitement imbriquées ${ }^{4}$. On admet généralement qu'à l'origine de ces deux mouvements, il y a entre autres un attachement aux qualités particulières de la nature, de certains lieux, de certains milieux et aussi une appréciation de la qualité de l'expérience de ces lieux, de la vie en ces lieux. Dès lors, on a voulu faire connaître et préserver ces lieux, cette nature et l'expérience qu'on y vivait. L'avènement de la science écologique et plus généralement des sciences de l'environnement est ensuite venu, beaucoup plus tard, offrir certaines bases scientifiques à ces convictions d'ordre plus expérientiel, philosophique, préscientifique et romantique. Les premières grandes pressions de l'exode rural et de l'urbanisation concomitante, associées à la révolution industrielle, ont fouetté les défenseurs de la nature, d'une éducation à la nature et d'une éducation plein air. À la suite d'un tel « recul de la nature ", selon l'expression de Bourg (1997), ont succédé des crises et des pénuries plus graves où la nature devenait essentiellement appréciée, connue et reconnue, pour les « ressources » qu'elle génère dans l'économie, qu'elle soit capitaliste ou socialiste. Ces crises ont stimulé le développement d'une éducation à la conservation, nettement plus ressourciste et utilitariste. À la fin des années 1960 et à l'orée des années 1970, l'internationalisation et la globalisation des perspectives et des problématiques environnementales, à la remorque de la mondialisation économique, politique et technologique, ont propulsé la création de ministères de l'Environnement et les développements plus formels d'une éducation relative à l'environnement. Ces développements furent aussi stimulés et orientés par une série de conférences onusiennes qui n'ont cessé de se multiplier et qui cherchent encore maintenant à baliser le parcours pour guider l'humanité et à définir des orientations pour son avenir, notamment en matière d'éducation.

9 Toutes ces visions, avec leur corollaire éducatif, existent encore maintenant. Dès lors, on ne sera pas surpris de constater qu'il puisse exister des tensions, parfois créatrices et parfois destructrices, entre divers points de vue, diverses conceptions, diverses théories et diverses pratiques en environnement et en éducation relative à l'environnement. Un premier de ces clivages possibles se manifeste entre les perspectives globales et locales, qu'un néologisme comme « glocal » ne peut totalement gommer, ou encore entre une perspective planétaire, internationale, nationale par rapport une perspective plus nettement locale, communautaire et incarnée dans un milieu bien précis et unique ou encore centrée sur un lieu. Un second clivage possible concerne la centration sur les problèmes environnementaux ou socioenvironnementaux, qu'on peut opposer, au moins partiellement, à une centration sur ce qui fonctionne bien dans l'environnement ou à une centration sur l'étude du milieu de vie sans agenda à priori, sauf peut-être celui de découvrir ou redécouvrir ce milieu. 
Un troisième clivage possible peut opposer une perspective plus historique, visant à comprendre la genèse de notre milieu de vie et de notre communauté afin de se les réapproprier et une perspective plus "futuriste " centrée plus radicalement sur un avenir à construire. Enfin, un quatrième clivage possible peut opposer une approche centrée sur les sciences et plus particulièrement certaines des sciences de la nature positivistes à des approches centrées sur d'autres champs du savoir ou à d'autres approches du savoir et de l'agir en ce qui concerne le rapport au monde : arts, lettres, histoire, philosophie et tous les savoirs non disciplinaires, incluant les "éco-savoirs " de notre inévitable "écoformation" (Moneyron 2001, 2003). Il ne s'agit là que de quelques clivages possibles et d'autres ont été proposés dans une recherche visant notamment à comparer une configuration d'appartenance à la nature à une configuration aliénante (Berryman, 2002a, 2003).

S'il peut être envisageable, dans certaines approches synthétiques, de réconcilier ces perspectives potentiellement antagonistes, il apparaît plus nettement qu'une perspective "ressourciste" internationale focalisant sur les grands problèmes environnementaux et sur les choix économiques, politiques et administratifs pour le futur de la planète semble dominer l'agenda environnemental et celui de l'éducation relative à l'environnement.

\section{Réenracinements : communauté et lieu}

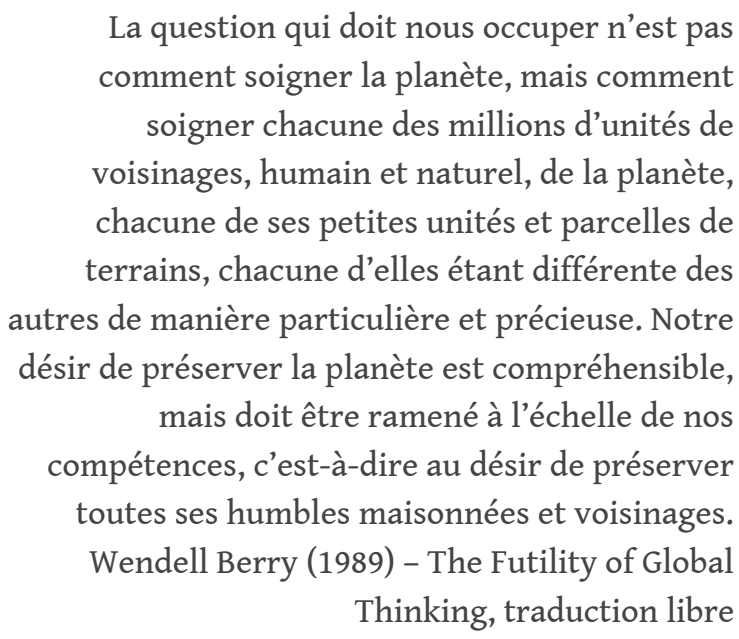

Sans prétendre trancher ou répondre de manière satisfaisante aux dilemmes et oppositions évoqués précédemment ou encore fournir une synthèse adéquate à ces tensions, clivages et ruptures, il y a des voix qui s'élèvent et des pratiques qui se mettent en place ou qui se redécouvrent autour d'une éducation relative à l'environnement centrée sur la communauté et sur les lieux. En anglais, elles ont des noms tels "bioregional education", "watershed education ", "place-based education", " place-based environmental education", "community education", "community-based environmental education » et " community and place-based environmental education ». Avant de chercher à caractériser ces approches, il est utile de s'arrêter aux termes. 


\section{Communauté}

Comme l'ont aussi noté Isabel Orellana (2002, p. 56) et Carine Villemagne (2002, p. 38), le mot « communauté " peut avoir diverses significations en français. Il en va de même en anglais bien que la langue anglaise semble tolérer dans ce cas une plus grande polysémie. En français, ou plus précisément en France, l'usage de communauté, en anglais "community", pour signifier "communauté locale», semble être plus généralement remplacé par les expressions « collectivité » ou " collectivité locale ».

Au Québec, l'usage du terme communauté est plus généralisé que celui de collectivité ou de collectivité locale. Le dictionnaire fait d'ailleurs des renvois entre ces deux mots. Pour l'écrivain étasunien et professeur d'anglais Scott Russel Sanders :

Les mots "communauté ", "communion» et «communiquer » dérivent tous de «commun», et les deux syllabes de "commun» proviennent de deux racines différentes, la première signifiant "ensemble» ou "à côté de", la seconde concernant le troc ou l'échange. Incarné dans ce mot, on retrouve le sens de notre vie partagée, une vie de réciprocité où l'on donne et l'on reçoit - musique, toucher, idées, recettes, histoires, soins, outils et tout le spectre d'artéfacts et de talents [...]. Une telle communauté ne naît pas du devoir ou de l'argent, mais d'échanges gratuits et libres entre des gens qui partagent un lieu, partagent le travail et la nourriture, la tristesse et les espoirs. Prendre part à la vie commune signifie d'habiter dans un réseau de relations. (Sanders, 1995, p. 79-80, traduction libre, emphase italique ajoutée)

Dans une perspective éducationnelle, la philosophe de l'éducation Nel Noddings (2005), en prenant appui sur les « éléments » de la communauté proposés par Selznick (1992), caractérise notamment la communauté de proximité ou de lieu par: l'identité (la reconnaissance), la participation (dans les fonctions de la communauté), l'historicité (la mémoire comme fondement de la communication), l'appartenance (le sentiment de sécurité), le mutualisme (l'assistance réciproque), l'ordre (provenant des pressions à la conformité) et l'intégration (socialisation dans un cadre offrant un point de vue intégré).

Plus largement, et écologiquement, l'intérêt du mot communauté consiste aussi dans le fait qu'il peut rappeler la notion de " communauté biotique » chère à l'écologiste Aldo Leopold (1982). Nous ne vivons pas exclusivement dans une communauté humaine, mais dans un ensemble plus large qui comprend aussi les autres êtres vivants. Une telle notion de communauté élargie est aussi inspirée de la notion de communauté en écologie, soit tous les organismes occupant un habitat commun. En lien direct avec le thème de lieu développé ici, rappelons que pour les écologistes ou les écologues, nous habitons un "biotope": un lieu «topos" de vie "bio». L'intérêt de la notion de communauté est de ne pas totalement se couper et s'aliéner de cette insertion dans la matrice du vivant. Pour l'écologiste et professeur canadien à la retraite John Livingston :

Un sentiment de communauté, à sa plus simple expression, est une conscience d' appartenance simultanée à une société et à un lieu et aussi une conscience d'une identité personnelle fondamentale en tant que cette société ou ce lieu. Il y a autant de sortes de sociétés et de lieux qu'il y a de sortes d'êtres vivants, et probablement tout autant de formes de consciences. Cependant, au niveau le plus fondamental, tout sentiment d'appartenance ne peut reposer que sur un sentiment de soi. Dès lors, notre quête d'un sentiment de communauté renouvelé dépendra finalement d'un sentiment de soi fort différent de celui avec lequel nous avons été endoctrinés. (Livingston, 1996, p. 132, traduction libre) 

qu'on enfermerait trop exclusivement dans un univers exclusivement humain. Il spécule alors, d'une manière que certains trouveront controversée, à l'effet que cette individualité exacerbée chez les êtres humains ne serait possiblement pas, contrairement à l'opinion répandue, un sommet de l'évolution atteint par les êtres humains, mais une sorte de stagnation à un sentiment plus primitif et plus enfantin alors que les autres animaux développeraient rapidement dans leur ontogenèse une meilleure identité communautaire et planétaire. Livingston s'appuie en partie sur la thèse voulant que notre évolution biologique se soit notamment faite par néoténie ou par juvénilisation. L'être humain conserverait les qualités juvéniles d'indétermination et c'est la culture qui détermine en bonne partie les suites de l'évolution des sociétés humaines ou, pour être plus exact, qui guide les orientations au monde de l'animal humain et corollairement des sociétés humaines. Il y aurait donc une forme de retrait partiel des dynamiques biologiques. Dans l'optique de cette thèse qui est paradoxalement à la fois culturaliste et sensible à notre biologie, la culture prend le relais et c'est à elle qu'incombe la tâche et la lourde responsabilité de construire la communauté, le sentiment de communauté, dont celui de l'appartenance à la communauté biotique. Or, nous nous couperions de plus en plus de cette communauté. Par contre, Livingston garde espoir et il fait en quelque sorte confiance en notre capacité de retrouver le sentiment de communauté biotique, de continuité avec le monde où nous baignons, en étant plus attentif à cette communauté qui existe bel et bien, malgré notre possible autisme et notre aveuglement. À titre d'exemple, rappelons-nous tout simplement qu'il $\mathrm{y}$ a encore cet extraordinaire "règne » végétal qui synthétise, à partir du gaz carbonique que nous expirons, l'oxygène que nous inspirons. Qu'on le veuille ou non, nous sommes en communauté biotique et la «flore bactérienne » de notre tube digestif peut nous le rappeler quotidiennement.

Par contre, on retiendra toutefois ici que lorsqu'il est question d'une éducation relative à l'environnement centrée sur la communauté, il est plus généralement question de se centrer sur la vie communautaire au sein de la collectivité locale. Bref, il est question d'œuvrer avec les gens de son milieu de vie. Observons ici que la communauté humaine ou la collectivité ne pourrait être le seul objet éducatif dans une perspective d'éducation relative à l'environnement. En effet, on serait là dans le champ qui caractérise l'éducation à la citoyenneté. Ainsi, l'un des intérêts de la notion de communauté est qu'elle laisse entrevoir une voie vers la communauté biotique, vers les autres êtres vivants, les végétaux et les autres animaux.

qui caractérise finalement la notion de communauté, est le fait de se centrer sur une certaine échelle, plus locale et sur une dynamique, avec les autres membres de la communauté, de la collectivité locale où l'on habite, de son milieu de vie, de son habitat, de son quartier. L'étape suivante est de passer de la reconnaissance de cette vie en commun avec d'autres êtres humains à une reconnaissance de notre communauté intime avec le vivant.

\section{Lieu}

Outre la communauté, il est aussi question d'une centration sur les lieux. Cette notion ancienne de lieu est évoquée lorsqu'il est question de l'esprit des lieux ou de "genius loci» (Norberg-Schulz, 1981). On parlera aussi du sens du lieu ou du «sense of place». 
Sans proposer une analyse fine des nombreux écrits sur la notion de lieu, notons seulement que plutôt qu'une focalisation sur des problèmes environnementaux ou sur une dynamique environnementale particulière, tels les changements climatiques ou le cycle de l'eau, il s'agit d'une centration sur des endroits particuliers du milieu de vie et sur l'expérience de tels lieux. Bref, il s'agit d'un ancrage prenant notamment appui sur une approche expérientielle d'endroits ou de localisations particulières du milieu de vie. Si un tel ancrage sur les lieux peut déboucher sur une prise en compte d'enjeux socio-environnementaux, ceux-ci ne constituent pas l'ancrage premier. De fait, nous sommes ici davantage dans la foulée de l'étude de milieu telle que Dehan et Oberlinkels (1984) l'envisagent et dans la foulée des approches d'écoformation (Cottereau, 2001). La notion de lieu comprend de fortes composantes expérientielles et affectives de « l'êtreau-monde" et de son "monde vécu». D'autres notions vers lesquelles certains cherchent aussi à orienter des pratiques éducatives, comme les notions de «bassin versant » et de "biorégion », pourraient possiblement s'associer à la notion de lieu. Toutefois, soulignons que les notions de bassin versant et de biorégion relèvent davantage de pratiques de gestion environnementale et qu'elles sont nettement plus objectives que la notion de lieu et qu'elles seront probablement associées à des pratiques éducatives plus scientifiques, rationnelles, positivistes. La centration sur les lieux et sur l'expérience des lieux correspond d'ailleurs à une partie de la conception de l'environnement chez Louis Goffin et chez Lucie Sauvé. Pour Louis Goffin (2001), l'environnement "désigne à la fois: 1) une réalité objectivable [...] 2) une problématique spécifique [...] 3) des modes stratégiques d'intervention pour prendre en charge les problèmes ». Cependant l'environnement désigne aussi :

4) Un champ psychosocial tel que le représente et le vit concrètement chaque individu plongé dans ses divers milieux de vie, personnels et partagés avec d'autres, avec lesquels il élabore et entretient un tissu de relations intimes (admiration, identification, aisance, malaise, fuite, imprégnation, découverte, compassions, transfert, identification, rejet, amour et affection, protection, soin, etc.) qui participent toujours à son développement, tantôt de manière positive, tantôt de manière plus négative. (Goffin, 2001)

Lucie Sauvé traite quant à elle de l'une des représentations sociales de l'environnement en tant que milieu de vie et l'une "des premières étapes d'éducation relative à l'environnement consiste à explorer et redécouvrir son propre milieu de vie, explorer l' ici et maintenant des réalités quotidiennes avec un regard neuf, appréciatif et critique à la fois » (Sauvé, 2002).

Le poète et fermier étasunien Wendell Berry, convaincu de la fécondité d'une attention plus soutenue aux lieux et inspiré notamment par les quelque trente dernières années de la vie de Jean Henri Fabre dans son petit hameau à Sérignan, avance que :

Ma propre expérience m'a montré qu'il est possible de vivre dans le même petit lieu et de l'étudier attentivement pendant plusieurs dizaines d'années consécutives et de réaliser que ce petit lieu échappe et dépasse sans cesse toute compréhension. Parce que ce lieu change continuellement et que ce changement demeure imprévisible, le lieu ne peut pas être cerné par une approche réductionniste. Un lieu, hormis notre capacité de le détruire, est inépuisable. Il ne peut pas être entièrement connu, vu, compris ou apprécié. (Berry, 2000, p. 139, traduction libre)

Berry se porte à la défense de l'étude et de l'enracinement dans un lieu, en lien avec les gens, pour habiter et soigner ce lieu, ces gens et cette communauté. Dans la même veine, Henry David Thoreau soutenait, il y a presque 150 ans, que : 
Mon voisinage offre plusieurs bonnes possibilités de promenades et même si pendant plusieurs années j'ai marché presque tous les jours et parfois plusieurs jours de suite, je n'ai pas épuisé ces possibilités. Un point de vue totalement nouveau est une grande source de joie et je peux en découvrir chaque après-midi. Deux ou trois heures de marche m'entraîneront dans un pays aussi étrange que je puisse imaginer. (Thoreau, 1862, p. 79, traduction libre)

Lieux et communautés offrent d'immenses possibilités ainsi que des contraintes. Ils contribuent aussi à définir des limites. Les articulations entre lieu et communauté sont réaffirmées par Wendell Berry quand il se porte à la défense des communautés locales par rapport aux intérêts plus économiques qui prônent la «liberté » de transactions internationales et financières de tout ce qui est ramené à du capital (et tout tend à être réduit en capital dans la logique économique dominante).

La communauté locale doit finalement se comprendre et se reconnaitre elle-même en tant que communauté d'intérêts - une dépendance commune par rapport à une vie commune et un lieu commun. Et parce qu'une communauté est par définition localisée («placed»), son succès ne peut être séparé du succès de son lieu, son milieu naturel et son environnement, ses sols, forêts, prairies, plantes et animaux, eau, lumière et air. (Berry, 1987a, p. 192, traduction libre)

\title{
Une éducation relative à l'environnement centrée sur la communauté et sur les lieux
}

\author{
Revenir aux choses mêmes, c'est revenir à ce \\ monde avant la connaissance dont la \\ connaissance parle toujours, et à l'égard duquel \\ toute détermination scientifique est abstraite, \\ signitive et dépendante, comme la géographie à \\ l'égard du paysage où nous avons d'abord appris \\ ce que c'est qu'une forêt, une prairie ou une \\ rivière. \\ Maurice Merleau-Ponty (1945, p. iii) - \\ Phénoménologie de la perception
}

Plusieurs écrits traitent de projets, de programmes et d'approches d'éducation relative à l'environnement prenant un certain appui sur la communauté ou sur les lieux, par exemple, l'approche "recherche-action : résolution de problèmes communautaires " (Bull et coll., 1988), le réseau ENSI Environment and School Initiatives, de l'OCDE (Mayer, 2003), le projet CADISPA Cooperation and Development in Sparsely Populated Areas (Robottom, 2003), le programme Éco-quartier (Villemagne, 2003) ou encore le Watershed Learning Center Program (Kenny, Price Militina et Horrocks Donohue, 2003). Chouinard et Pruneau (1999) font appel à la notion de développement de communautés responsables. Traina et Darley-Hill (1995) invitent à une éducation biorégionale. Pivnick (2003) vise à ré-ancrer ses errances doctorales dans une "approche écologique de recherche » via une attention plus soutenue au lieu. Notons aussi que deux manuels récents traitent d'éducation relative à l'environnement centrée sur la communauté et sur les lieux, l'un plus nettement stratégique a été produit par David Sobel (2004) en se basant notamment sur l'expérience développée dans une université de la Nouvelle-Angleterre, l'autre, plus nettement pédagogique a été rédigé par Lucie Sauvé et ses collaboratrices (2001) en se basant notamment sur un projet de coopération internationale en Amazonie. Enfin, David Orr (1994) propose, quant à lui, qu'à tous les niveaux, du 
préscolaire au doctorat, une partie du curriculum soit centrée sur l'étude expérientielle de l'environnement local et particulièrement l'environnement naturel local.

\section{Quelques caractéristiques}

Lucie Sauvé et ses collaboratrices, alors qu'elles traitent de «l'approche communautaire ", rappellent que :

L'école n'est pas isolée, elle s'inscrit dans un milieu de vie partagé. Elle fait partie intégrante d'une réalité communautaire caractérisée par sa culture spécifique. Avec des projets pédagogiques impliquant l'exploration, la recherche, l'amélioration ou la transformation du milieu, l'école peut jouer un rôle clé dans le développement de la communauté, surtout si elle associe à ses projets diverses personnes et divers organismes du quartier ou du village. [...] La création de liens avec la communauté favorise le développement d'un sentiment d'appartenance à cette dernière. De cette relation peut naître un désir d'engagement collectif dans un processus de changement pour une meilleure qualité de vie. (Sauvé et coll., 2001, p. 44)

Pour David Sobel, enseignant et chercheur à la faculté d'éducation du Antioch New England Graduate School :

L'éducation relative à l'environnement centrée sur les lieux est un processus éducatif utilisant la communauté locale ainsi que l'environnement local en tant qu'entrées pour l'enseignement des langues, des mathématiques, des sciences humaines, des sciences ainsi que pour les autres objets du curriculum. En mettant l'accent sur les approches expérientielles et concrètes du monde réel, cette approche améliore le rendement scolaire, aide les élèves à développer des liens plus forts avec leur communauté, accroît l'appréciation du monde naturel chez les élèves et crée un engagement accru à servir la communauté en tant que citoyen actif.

Corollairement, la vitalité de la communauté et les qualités de l'environnement se trouvent accrues par l'engagement et la participation active, de citoyens de la localité, des organisations communautaires et des expertises locales en matière d'environnement, dans la vie de l'école. (Sobel, 2004, p. 7, traduction libre)

Pour Delia Clark (2002), collègue de David Sobel, l'éducation relative à l'environnement centrée sur les lieux peut contribuer à «revitaliser la communauté». Ses «six ingrédients de base » sont :

1. L'enracinement dans l'environnement local, la communauté locale et le patrimoine local.

2. Les partenariats soutenus et intensifs: école, organisations communautaires et centres d'éducation relative à l'environnement.

3. L'engagement continu au sein de la communauté: liens entre préoccupations sociales et environnementales, expérience et utilisation de ressources en dehors de l'école, etc.

4. La pédagogie de projet : travailler sur des besoins authentiques, œuvrer à des projets en lien avec l'âge et le niveau des participants, etc.

5. Le développement professionnel et la formation continue des enseignants et éducateurs.

6. L'évaluation formative. (Clark, 2002, traduction libre)

Selon Woodhouse et Knapp (2000), les caractéristiques de l'éducation centrée sur les lieux sont les suivantes :

1. Elle émerge fondamentalement des qualités particulières d'un lieu.

2. Elle est fondamentalement multidisciplinaire. 
3. Elle est fondamentalement expérientielle. Dans plusieurs programmes, ce volet expérientiel comprend une participation à la vie de la communauté ainsi que des services à la communauté.

4. Elle reflète une vision de l'éducation qui va au-delà de la qualification professionnelle ou de l'acquisition de compétences pour un futur emploi.

5. Elle relie le lieu avec soi et avec la communauté. La perspective écologique met ces liens en évidence. Ces liens lieu-soi-communauté impliquent par ailleurs des dimensions multigénérationnelles et multiculturelles. (Woodhouse et Knapp, 2000, traduction libre) lieux et la communauté, l'Agence de protection de l'environnement des États-Unis (EPA) propose cette conception de l'éducation relative à l'environnement centrée sur la communauté :

L'éducation relative à l'environnement centrée sur la communauté :

- Est en lien direct avec des sujets, des problèmes ou des enjeux locaux.

- Fournit des actions pratiques en lien avec les besoins identifiés par des individus ou des groupes et qui peuvent être effectuées de manière réaliste.

- Résulte en des actions de gestion environnementale qui émergent de la communauté plutôt que depuis des exigences dirigées vers la communauté. (United States Environmental Protection Agency, 1998, traduction libre)

Particulièrement à l'aune de ce dernier exemple, on constate qu'il peut exister des clivages entre une centration sur les lieux et une centration sur la communauté. Ainsi, dans l'exemple ci-dessus, l'EPA étasunien, une agence nationale avec un agenda national, veut finalement malgré tout utiliser la communauté locale pour résoudre des problèmes environnementaux qui relèvent de son propre agenda d'agence nationale. On pourrait ici remplacer l'EPA par d'autres agences internationales, nationales ou régionales qui doivent dorénavant accepter de travailler avec des communautés afin d'accomplir leur mandat. Dans de tels cas, la perspective éducative est possiblement plus instrumentale et risque d'être mise au service de la résolution de problèmes. Il y a de possibles dérives où la communauté pourrait même devenir littéralement un nouvel instrument pour un agenda exogène qui lui échapperait.

\section{L'exemple « CO-SEED »}

Voici une approche plus endogène visant à développer une éducation relative à l'environnement centrée sur la communauté et sur les lieux qui soit spécifique à chaque école, à chaque communauté, à chaque lieu. Dans le manuel Place-Based Education, David Sobel traite de l'initiative "Community-based School Environmental Education» (CO-SEED) dans laquelle il est engagé depuis six ans. On pourrait traduire l'acronyme CO-SEED par l'acronyme Scol-ERE qui signifierait «Synergie Communauté et Lieu en Éducation Relative à l'Environnement ». L'analogie avec la graine, "seed", souligne une visée de développement organique ou endogène en étant à la recherche d'un sol fertile où semer et faire germer la graine. Sobel et ses collègues de la faculté d'éducation du New England Graduate School œuvrent à une échelle locale, micro, école par école. Le modèle qu'ils ont élaboré mène à un travail en profondeur sur un site plutôt qu'à un grand programme national et thématique.

Sobel et ses collègues sont donc à l'affût de sites potentiellement fertiles où peuvent se combiner : a) un intérêt manifeste pour l'étude du milieu, b) un support administratif

Éducation relative à l'environnement, Volume 5 | 2005 
manifeste au sein des organisations impliquées, c) un groupe d'enseignants engagés, d) un dialogue école et communauté et e) la disponibilité d'une organisation spécialisée ou d'une structure qui peut soutenir des apprentissages en matière d'environnement et de relations à l'environnement telle qu'un centre d'interprétation, un musée ou une société d'histoire (Sobel, 2004, p. 49-52). Le travail de longue haleine consiste à établir des liens entre l'école et la communauté, à transformer le curriculum pour l'adapter aux réalités locales et à soutenir les relations synergiques entre les acteurs engagés.

Le travail des formateurs universitaires consiste à formaliser la mise en place d'une dynamique école et communauté qui se penchera en quelque sorte sur l'esprit des lieux et y localisera le curriculum. Ce travail se fait par une équipe mixte dans un partenariat entre universités, commissions scolaires, organisations communautaires et organisations spécialisées pour soutenir des apprentissages en matière d'environnement. La communauté se définit alors des priorités et les écoles s'engagent dans des projets réels centrés sur le milieu de vie, en lien avec la communauté.

Dans une optique presque complémentaire à l'orientation du manuel plutôt stratégique de David Sobel, rappelons que Lucie Sauvé et ses collaboratrices (2001, p. 16-17) proposent un manuel plus pédagogique visant à renforcer les liens école et communauté. Ce manuel de formation propose diverses activités visant à :

1. Redécouvrir son propre milieu de vie.

2. Explorer son quartier ou son village.

3. Prendre conscience du réseau des interactions entre les éléments du milieu ainsi qu'entre ces éléments et soi-même, en tant qu'individu et groupe social.

4. Découvrir et diagnostiquer des situations problèmes.

5. Se pencher sur le processus de résolution de problèmes environnementaux.

6. Mettre en place des projets d'amélioration de la qualité du milieu de vie.

\section{Apprendre à habiter ici et entre nous}

En quoi les caractéristiques émanant de ces définitions et pratiques permettent-elles de différencier l'éducation relative à l'environnement centrée sur la communauté et sur les lieux d'autres approches?

Premièrement, la centration sur la communauté locale contraste avec de nombreuses approches d'éducation relative à l'environnement qui pourraient être totalement indépendantes de cette communauté, par exemple : demeurer à l'intérieur de la classe, être en lien ou faire des échanges avec des élèves d'autres pays ou encore être en lien avec des professions ou des ordres professionnels, sans lien étroit avec les membres de sa communauté.

Soulignons toutefois que de telles approches indépendantes de la communauté peuvent s'avérer pertinentes selon le contexte où elles sont adoptées. Dans le cas de la centration sur la communauté, il y a donc un maillage avec les autres habitants du milieu de vie des élèves. Il y a des ouvertures mutuelles entre école et communauté. Nous ne sommes toutefois pas ici dans l'impératif si souvent entendu que «l'école doit s'ouvrir au milieu». Plus simplement, école et communauté découvrent qu'elles partagent un lieu, un milieu, qu'elles s'y insèrent et s'y inscrivent.

Deuxièmement, la centration sur le milieu de vie et les lieux spécifiques se démarque, au départ, d'autres approches: disciplinaires (géographie, écologie, par exemple), 
thématique (eau, air, animaux, biodiversité, énergie, etc.), résolutique (problèmes environnementaux ou autres et leurs solutions: changements climatiques, récupération et recyclage, «Action 21 », etc.). L'approche se veut donc beaucoup plus endogène qu'exogène, sans un agenda externe prédéterminé, sans une proposition thématique externe ou sans programme. On est davantage dans le ici et maintenant entre nous. C'est le travail sur la question « qu'est-ce qu'il y a ici? » de Wendell Berry (1987b, p. 146). «L'esprit du lieu » peut toutefois ouvrir sur différentes thématiques, les révéler, les contextualiser ou encore les ancrer, leur donner vie. Ces émergences thématiques associées à l'expérience du lieu peuvent devenir objets de projets personnels et communautaires.

Troisièmement, une approche par le lieu devrait forcément avoir une composante expérientielle ou phénoménologique puisque la notion même de lieu fait en quelque sorte appel au monde vécu, à l'être-au-monde. Le lieu ne devrait pas se chosifier. Comme le souligne l'anthropologue Michael Jackson, « le sentiment d'être chez soi dans le monde est moins ancré dans le lieu en tant que tel que dans les activités qui se déroulent dans ce lieu » (2000, p. 148). Cette approche invite donc à une éducation formelle en dehors de la salle classe avec ce que cela comprend comme lot de risques et de plaisirs comme en témoignent Crimmel (2003), Sobel (1993) ainsi que Leslie, Tallmadge et Wessels (1999).

41 Évidemment, il y a des limites et des difficultés associées à une éducation relative à l'environnement prenant appui sur les lieux et la communauté. Des difficultés réelles ou potentielles ont été évoquées: domination des hiérarchies ou des élites locales, collectivités où il y un très faible sentiment de communauté, communautés étouffantes et milieux de vie aux qualités socio-environnementales douteuses, par exemple à cause de violences, de pollutions ou de manque de diversité (Berryman, 2004). Laurent Marty (2004) évoque les difficultés avec des lieux moroses et des communautés moroses. On pourrait aussi craindre, au premier abord, une sorte d'hyperlocalisme avec un fort esprit de clocher et même un certain " tribalisme ».

Mais on peut tout aussi bien concevoir l'universalisme globalisant comme une forme d'impérialisme, de tribalisme "occidentalocentrique » et "économiciste " (Latouche, 2000) qui vise à imposer sa propre cosmologie, sa vision du monde et ses totalisations aux autres. D'ailleurs les grands conflits mondiaux n'ont-ils pas souvent à la base des visées d'universalisation hégémonique de l'une des parties qui cherche à s'imposer ? De plus, il faut aussi reconnaître que les dimensions planétaires des techno-sciences nous pénètrent déjà de part en part et ce n'est certes pas d'hyper-localisme dont nous semblons souffrir. Dans une perspective éducative, Phillip Payne $(2003 a, b)$ propose une analyse critique de l'intensification de l'envahissement de notre monde vécu par les technologies. Pensons seulement aux changements fulgurants de notre être-au-monde via les ordinateurs qui meublent nos vies et prennent en charge une bonne partie de notre expérience depuis seulement 10 à 20 ans et qui nous invite souvent, virtuellement, à "visiter » des "sites ». Il y a bien entendu la télévision qui nous " planétarise » à sa manière depuis une cinquantaine d'années. L'univers marchand du capital mondialisé nous sollicite sans cesse afin de se renouveler. Dans les grandes villes, la présence des panneaux publicitaires et de réclames de toutes sortes est envahissante. Les publicitaires ne cessent de chercher de nouvelles manières et de nouveaux lieux pour nous solliciter afin que le capital charme le «capital humain » et qu'il se reproduise. Les journaux sont remplis de publicités. Ainsi, pour la plupart 
d'entre nous, nous sommes bien davantage exposés à un discours politique et économique mondial plutôt qu'aux réalités de nos milieux de vie. C'est "l'occidentalocentrisme» triomphant avec son «économicisation» et sa «marchandisation " du monde qui nous font glisser vers une "planète uniforme » (Latouche, 2000).

Une démonstration saisissante de cet envahissement de nos vies a été faite à Montréal. Dans un court film, Laure Waridel et Hugo Latulipe montrent comment les jeunes des écoles secondaires parviennent facilement à nommer chacune de grandes entreprises lorsqu'on leur présente les différents logos alors qu'ils sont incapables de nommer des oiseaux assez communs lorsqu'on leur présente des images de ceux-ci ${ }^{5}$. Symboliquement et matériellement, notre vie, notre milieu de vie et notre culture sont traversés de mondialisations marchandes. Dans ce contexte, il ne faut pas craindre une hypersensibilité au lieu, mais plutôt un oubli de celui-ci. Il pourrait donc sembler plus impératif de redécouvrir notre milieu de vie et de réapprendre à y habiter, car nous habitons toujours quelque part. Pour la plupart, nous ne souffrons certainement pas d'un sur-engagement individuel et communautaire dans l'étude de nos milieux de vie. À cet égard, les défis éducatifs sont importants : il s'agit de réinscrire notre être dans le monde, en lien avec ces lieux qui continuent de nous porter et avec ces communautés où nous sommes, contribuant à notre formation et contribuant à renforcer notre identité personnelle et collective. D'ailleurs, dans ses réflexions plus récentes sur le bonheur et l'éducation, Nel Noddings (2005), dont les écrits en philosophie de l'éducation sont associés à l'éthique de la sollicitude et au féminisme, traite entre autres des pratiques éducatives portant sur les liens avec la nature, les liens avec les lieux et les liens avec la communauté. Elle associe ces pratiques avec quelques autres pierres d'assises d'un bonheur auquel l'éducation scolaire devrait contribuer. Rappelons qu'on associe plus rarement éducation formelle et bonheur. Néanmoins, que l'éducation soit intimement associée au bonheur pourrait être autre chose qu'une utopie (du grec «ou» non, et «topos» lieu, en aucun lieu) et pourrait au contraire s'incarner dans l'expérience de la nature, du lieu et de la communauté.

\section{BIBLIOGRAPHIE}

Berger, P.L. et Luckmann, T. (1966). The Social Construction of Reality : A Treatise in the Sociology of Knowledge. New York : Doubleday \& Anchor.

Berry, W. (1987a). Does Community Have a Value ? Dans Berry, W. (dir.), Home Economics : Fourteen Essays by Wendell Berry (p. 179-192). San Francisco : North Point Press.

Berry, W. (1987b). Preserving Wildness. Dans Berry, W. (dir.), Home Economics : Fourteen Essays by Wendell Berry (p. 137-151). San Francisco : North Point Press.

Berry, W. (1989). The Futility Of Global Thinking. Harper's. Septembre 1989, p. 16-22.

Berry, W. (2000). Life is a Miracle : An Essay Against Modern Superstition. Washington : Counterpoint. 
Berryman, T. (1990). La conservation des qualités de la montagne. Mémoire présenté au Bureau de consultation de Montréal lors de la consultation publique sur le plan de mise en valeur du mont Royal. Document non publié.

Berryman, T. (1993). La conservation des qualités sauvages de l'espace naturel du Collège Jean-de-Brébeuf et le développement des institutions de savoirs. Mémoire présenté au Comité conseil d'arrondissement Côte-des-Neiges \Notre-Dame-de-Grâce. Document non publié.

Berryman, T. (1997). L'éducation relative à l'environnement : un nom récent pour une longue tradition. Sur la montagne, 13(1),4-8.

Berryman, T. (1998). L'avenir viable, un développement insoutenable en éducation : Dix motifs pour s'y objecter et défendre les qualités de l'éducation et des relations au monde. Communication présentée dans le cadre de la «Journée de discussion sur les enjeux contemporains de l'éducation relative à l'environnement », 17 juin 1998, Université du Québec à Montréal.

Berryman, T. (1999). Relieving Modern Day Atlas of an Illusory Burden : Abandoning the Hypermodern Fantasy of an Education to Manage the Globe. Canadian Journal of Environmental Education, 4, 50-68.

Berryman, T. (2002a). Éco-ontogenèse et éducation : les relations à l'environnement dans le développement humain et leur prise en compte en éducation relative à l'environnement durant la petite enfance, l'enfance et l'adolescence. Mémoire de maîtrise en éducation, Université du Québec à Montréal.

Berryman, T. (2002b). Printemps silencieux + 40. Int'ERE.net,1(1), Mars 2002. Version en ligne sur http://www.aqpere.qc.ca/intereb.15.03.02.html.

Berryman, T. (2002c). What is Here ? Some Foundations to Envision Community and Place Based Environmental Education. EECOM News, August 2002. Version en ligne http://www.eecom.org/ francais/comere_03.html.

Berryman, T. (2003). L'éco-ontogenèse : les relations à l'environnement dans le développement humain - d'autres rapports au monde pour d'autres développements. Éducation relative à l'environnement : Regards - Recherches - Réflexions, 4, 207-228.

Berryman, T. (2004). Caractéristiques, portée et limites de l'éducation relative à l'environnement centrée sur la communauté et sur les lieux. Communication présentée au Colloque Environnements, cultures et développements, 7 et 8 novembre 2002, Ifrée-ORE, Niort, France.

Berryman, T, et Dumouchel, C. (2002). Communaut'ERE : les défis de l'éducation relative à l'environnement centrée sur la communauté. Une tentative de synthèse critique. Communication présentée dans le cadre de la huitième conférence annuelle du réseau canadien d'éducation et de communication relative à l'environnement (EECOM), «Communaut'ERE : les défis de l'éducation relative à l'environnement centrée sur la communauté », 11-15 août 2002, Montréal.

Bertrand, Y. et Valois, P. (1999). Fondements éducatifs pour une nouvelle société. Montréal / Paris : Éditions Nouvelles / Chronique Sociale.

Bourg, D. (1997). Nature et technique : essai sur l'idée de progrès. Paris : Hatier, Collection « Optiques philosophie » no. 220.

Boutin, G. et Julien, L. (2000). L'obsession des compétences : son impact sur l'école et la formation des enseignants. Montréal : Éditions nouvelles, Collection « Éducation et Éducation spécialisée ».

Bull, J., Cromwell, M., Cwikiel, W., Di Chiro, G., Guarino, J., Rathje, R., Stapp, W., Wals, A.E., et Youngquist, M. (1988). Education in Action: A Community Problem Solving Program for Schools. Dexter, MI : Thomson-Shore. 
Chouinard, O. et Pruneau, D. (1999). La notion de développement de communautés responsables comme source d'inspiration pour la recherche en éducation relative à l'environnement (en collaboration avec Djibo Boubacar). Canadian Journal of Environmental Education, 4, 142-154.

Clark, D. (2002). Community Vitality Through Place-Based Education. Communication présentée dans le cadre de la huitième conférence annuelle du réseau EECOM «Communaut'ERE : les défis de l'éducation relative à l'environnement centrée sur la communauté », 11-15 août 2002, Université du Québec à Montréal.

Cottereau, D. (2001). Pour une formation écologique : complémentarité des logiques de formation. Éducation permanente, 148, 57-67.

Crimmel, H. (dir.) (2003). Teaching in the Field: Working with Students in the Outdoor Classroom. Salt Lake City : University of Utah Press.

Dehan, B. et Oberlinkels, J.P. (1984). École et milieu de vie, partenaires éducatifs - Une pédagogie de projet interdisciplinaire. Cladech : Centre interdisciplinaire de recherches et d'applications pour le développement d'une éducation en milieu de vie (CIRADEM).

Dumont F. (1968). Le lieu de l'Homme. Montréal : HMH.

Emerson, R.W. (1856). Essays and English Traits. New York : P.F. Collier and Son.

Goffin, L. (2001). L'éducation relative à l'environnement (ERE) : conceptions, publics cibles, acteurs et stratégies. Dans Debuyst, F., Defourny, P et Gérard, H. (dir.), Savoirs et jeux d'acteurs pour des développements durables (p. 314-322). Louvain-la Neuve / Paris : Bruylant Academia (Collection « Population et Développement » no. 9) / L'Harmattan.

Jackson, M. (2000). At Home in the World. Londres et Durham : Duke University Press (originalement publié en 1995).

Kenny, J., Price Militina, H. et Horrocks Donohue, M. (2003). Helping Teachers to Use Their School's Backyard as an Outdoor Classroom : A Report on the Watershed Learning Center Program. Journal of Environmental Education, 35(1), 18-26.

Latouche, S. (2000). La planète uniforme. Castelnau-le-Lez : Climats, Collection « Sisyphe » (originalement publié en italien en 1997 sous le titre Il pianeta uniforme : Significato, portata e limiti dell'occidentalizzazione del mondo).

Laval, C. et Weber, L. (2002). Le nouvel ordre éducatif mondial : OMC, Banque mondiale, OCDE, Commission Européenne. Paris : Éditions Nouveaux Regards et Éditions Syllepse.

Leopold, A. (1982). A Sand County Almanac : With Essays on Conservation From Round River. New York : Ballantine Books (originalement publié en 1949).

Leslie, C. W., Tallmadge, J. et Wessels, T. (1999). Into the Field : A Guide to Locally Focused Teaching. Great Barrington, MA : The Orion Society.

Lévi-Strauss, C. (2003). Ramener la pensée à la vie. (Entretien avec Georges Kutukdjian). Magazine littéraire, 5, 53-59 (originalement publié en 1971).

Livingston, J.A. (1996). Other Selves. In Vitek, W. et Jackson, W. (dir.), Rooted in the Land : Essays on Community and Place (p. 132-139). New Haven, CT : Yale University Press.

Marty, L. (2004). Esprit des territoires es-tu là ? Lieux moroses et lieux vivants. Communication présentée au Colloque Environnements, cultures et développements, 7 et 8 novembre 2002, IfréeORE, Niort, France. 
Mayer, M. (2003). L'expérience du réseau ENSI de l'OCDE : L'école comme modèle de communauté apprenante. Éducation relative à l'environnement : Regards - Recherches - Réflexions, 4, 269-281.

Merleau-Ponty, M. (1945). Phénoménologie de la perception. Paris : Gallimard.

Moneyron, A. (2001). Vers une alternance écosociologique : écosavoirs des bergers transhumants. Éducation permanente, 148, 267-277.

Moneyron, A. (2003). Transhumance et Éco-Savoir : reconnaissance des alternances écoformatrices. Paris : L'Harmattan.

Noddings, N. (2005). Happiness and Education. Cambridge, UK : Cambridge University Press (originalement publié en 2003).

Norberg-Schulz, C. (1981). Genius loci : paysage - ambiance - architecture. Bruxelles : Pierre Mardaga éditeur.

Orellana, I. (2002). La communauté d'apprentissage en éducation relative à l'environnement : signification, dynamique, enjeux. Thèse de doctorat en éducation, Université du Québec à Montréal. Orr, D. (1994). Earth in Mind : On Education, Environment and the Human Prospect. Washington, DC : Island Press.

Ortega Y. Gasset, J. (1914). Meditaciones del Quijote. Madrid : Ediciones Catedra S.A. Payne, P. (2003a). Postphenomenological Enquiry and Living the Environmental Condition. Canadian Journal of Environmental Education, 8, 169-190.

Payne, P. (2003b). The Technics of Environmental Education. Environmental Education Research, $9(4), 525-541$.

Petrella, R. (2000). L'éducation victime de cinq pièges. Montréal : Éditions Fides.

Pineau, G. (1999). La formation expérientielle en auto-, éco- et co-formation. Éducation permanente, 100/101, 23-30.

Pivnick, J. (2003). In Search of an Ecological Perspective to Research : A Meditation on Topos. Canadian Journal of Environmental Education, 8, 143-154.

Robottom. Ian. (2003). Communautés, enjeux environnementaux et recherche en éducation relative à l'environnement. Éducation relative à l'environnement : Regards - Recherches - Réflexions, 4 , 77-95.

Sanders, S.R. (1995). Writing from the Center. Bloomington et Indianapolis : Indiana University Press.

Sauvé, L. (2002). L'éducation relative à l'environnement : possibilités et contraintes. Connexion, 23(1-2), 1-4.

Sauvé, L. et Berryman, T. (2001-2002). Contre de nouvelles aliénations. Politis, 34. Version en ligne. Sauvé, L., Berryman, T. et Brunelle, R. (2003). Environnement et développement : la culture de la filière ONU. Éducation relative à l'environnement : Regards - Recherches - Réflexions, 4, 33-55.

Sauvé, L., Orellana, I., Qualman, S. et Dubé, S. (2001). L'éducation relative à l'environnement : école et communauté : une dynamique constructive : guide de pratique et de formation. Montréal : Hurtubise HMH.

Selznick, P. (1992). The Moral Commonwealth : Social Theory and the Promise of Community. Berkeley : University of California Press. 
Sobel, D. (1993). Children's Special Places : Exploring the Role of Forts, Dens and Bush Houses in Middle Childhood. Tucson, AZ : Zephyr Press.

Sobel, D. (2004). Place-Based Education : Connecting Classrooms and Communities. Great Barrington, MA : The Orion Society.

Thoreau, H.D. (1862). Walking. In Elder, J. (dir., 1991), Nature/Ralph Waldo Emerson and Walking/ Henry David Thoreau (p. 69-122). Boston : Beacon Press.

Traina, F. et Darley-Hill S. (1995). Perspectives in Bioregional Education. Troy : North American Association for Environmental Education (NAAEE).

United States Environmental Protection Agency. (1998). An EPA/USDA Partnership to Support Community-Based Education : Discussion Paper. Washington DC : United States Environmental Protection Agency, Office of External Affairs, EPA 910-R-98-008 (non paginé).

Villemagne, C. (2002). Le programme d'action environnementale "Éco-quartier»: Propositions pour l'élaboration d'un cadre conceptuel et théorique. Montréal : Chaire de recherche du Canada en éducation relative à l'environnement, Université du Québec à Montréal (UQAM).

Villemagne, C. (2003). Le Programme d'action environnementale Éco-quartier de Montréal : Quel(s) environnement(s) et quelles culture(s) pour quel(s) développement(s) en contexte d'éducation communautaire ? Éducation relative à l'environnement : Regards - Recherches - Réflexions, $4,113-128$.

Woodhouse, J.L. et Knapp, C.E. (2000). Place-Based Curriculum and Instruction : Outdoor and Environmental Education Approaches. Washington DC : ERIC Clearinghouse on Rural and Small Schools (ERIC Digest EDO-RC-00-6).

\section{NOTES}

1. Il importe de souligner que le thème de cet article a aussi été traité dans des communications et des écrits associés à d'autres contextes et qu'ils appartiennent néanmoins à la gestation et à la genèse du présent texte. Ce thème apparaît dans des approches phénoménologiques afin de défendre un lieu public, le mont Royal à Montréal, et de défendre l'expérience de cette montagne face à des projets de développement (Berryman, 1990, 1993). Il apparaît aussi dans un résumé de l'histoire de l'éducation relative à l'environnement (Berryman, 1997). Dialectiquement, ce thème ressurgit à la suite du forum Planèt'ERE 1 dans une virulente critique de l'éducation au développement durable (Berryman, 1998), pour être ensuite reformulé dans une résistance plus constructive (Berryman, 1999). À l'aube du forum Planèt'ERE 2, un regard similaire est porté à l'invite du périodique Politis (Sauvé et Berryman, 2001-2002). Au printemps 2002, la tapageuse approche du Sommet de la Terre de Johannesburg (Rio +10), devient une occasion de réitérer un attachement aux lieux (Berryman, 2002b). L'organisation de la huitième conférence annuelle du Réseau canadien d'éducation et de communication relatives à l'environnement (EECOM) offre l'occasion de soutenir le choix du thème des lieux et de la communauté pour la conférence (Berryman, 2002c) et de faire une synthèse critique de celle-ci (Berryman et Dumouchel, 2002). Une nouvelle tentative de synthèse sur ces questions est formulée pour le colloque « Environnements, cultures et développements » organisé par l'Ifrée (Berryman, 2004).

2. Les réponses à ces questions sont l'une des clefs qu'utilisent entre autres Yves Bertrand et Paul Valois (1999) afin de caractériser différents paradigmes socioculturels et éducationnels.

3. La notion de dé-réfication est empruntée aux sociologues Berger et Luckmann (1966, p. 90). La réification consiste à chosifier une construction sociale ou une réalité sociale. Elle serait une tendance assez «naturelle » ou spontanée. La dé-réification est une forme d'exercice visant à 
resituer ce qui est pris comme objet, comme fait irréfutable ou comme réalité objective, dans la sphère du construit social.

4. Notons aussi que ces histoires imbriquées de l'environnementalisme et de l'éducation relative à l'environnement sont aussi étroitement imbriquées à l'histoire et à la mouvance du monde de l'éducation. Ce dernier serait quant à lui de plus de plus en plus instrumental et soumis à des impératifs et à des règles (nomos) « éco-nomique » avec une faible préoccupation " éco-logique ». Voir par exemple dans Boutin et Julien (2000), Petrella (2000), Laval et Weber (2002) ainsi que Sauvé, Berryman et Brunelle (2003).

5. Ce film fut présenté le $1^{\mathrm{er}}$ mai 2002 lors de l'événement joliment nommé « Revenons sur Terre », organisé par la Commission scolaire de Montréal.

\section{RÉSUMÉS}

Dans une perspective philosophique et historique, cet article débute en soulignant l'intérêt de renouer avec des pratiques éducatives où les voies d'entrée se font à partir de croisements entre lieu et communauté. L'intérêt de telles entrées bien localisées s'explique notamment à l'aune d'un possible désenchantement face à une voie d'entrée relativement similaire, mais plus planétaire, totalisante et globalisante : la « communauté » de tous les êtres humains d'un « lieu » gigantesque, la planète. La critique des émergences totalisantes invite à de nécessaires et difficiles réenracinements. Ce texte propose quelques éléments de clarification des notions de communauté et de lieu. À partir de ces ancrages, l'article explore la notion d'éducation relative à l'environnement centrée sur la communauté et sur les lieux, d'abord à partir de définitions puis à partir de certaines pratiques. Ceci permet de souligner des contrastes avec d'autres approches d'éducation relative à l'environnement.

From a philosophical and historical perspective, this paper begins by stressing the interest of renewing with educational approaches focusing on place and community. This arises partly in response to disenchantment with regards to approaches focusing on a large and most often planetary scale. Criticizing the contemporary trends towards globalizing and totalizing approaches invites to some alternative grounding for environmental education. Accordingly, this text proposes some clarifications of the notions of community and place and explores the notion of community and place-based environmental education, first through definitions and then through examples of practice. This finally allows to contrast community and place-based environmental education with other approaches to environmental education.

\section{AUTEUR}

\section{TOM BERRYMAN}

Titulaire d'un baccalauréat en biologie et d'une maîtrise en éducation, il prépare un doctorat en éducation portant sur l'éducation relative à l'environnement dans la formation initiale à l'enseignement primaire. Il est chercheur à la Chaire de recherche du Canada en éducation relative à l'environnement où il participe à divers projets de recherche et activités de formation. Il a œuvré pendant de nombreuses années à Montréal, dans un centre d'éducation relative à 
l'environnement au mont Royal, au sein de l'Association québécoise pour la promotion de l'éducation relative l'environnement (AQPERE) et à titre de travailleur autonome. 